\title{
INFLUENCE OF ROTOR DOWNWASH ON VERTICALLY DISPLACED NANOBOTS IN FLIGHT
}

\author{
Patrick P. NEUMANN ${ }^{1}$, Paul HIRSCHBERGER ${ }^{1}$, Matthias BARTHOLMAI ${ }^{1}$ \\ 1 Bundesanstalt für Materialforschung und -prüfung (BAM), Unter den Eichen 87, Berlin, Germany, \\ E-mail: patrick.neumann@bam.de
}

\section{Introduction}

Using a swarm of copter-based gas-sensitive aerial nano robots for monitoring indoor air quality is challenging due to, e.g., limited air space in buildings. To avoid an over-regulation of the available indoor air space (e.g., prohibit copters to fly above each other), a safety region around each copter must be defined to guarantee a safe operation of the swarm.

The key contributions of this paper are the realization of experiments that investigate the influence of the rotor downwash on flying vertically displaced nano robots and the development of a model describing the above-mentioned safety region.

\section{Robotic Platform}

The Crazyflie 2.0 (Bitcraze AB, Sweden) is a flying open-source development platform based on a quadrocopter (Fig. 1, in the following also referred to as nano UAV) [1]. With a take-off weight of $27 \mathrm{~g}$ it achieves flight times of up to 7 minutes. The four DC-motors allow a maximum takeoff weight of $42 \mathrm{~g}$, i.e., approx. $15 \mathrm{~g}$ remains for additional payload. The diameter of the platform is less than $0.1 \mathrm{~m}$. The nano UAV has a 10 degrees of freedom Inertial Measurement Unit (IMU) and an expansion connector gives access to important interfaces such as $\mathrm{I}^{2} \mathrm{C}$, UART, and SPI, as well as PWM, analog in/out, and GPIO. A $2.4 \mathrm{GHz}$ data and control link is used for sending control commands and receiving telemetry and payload data. Via Robot Operating System (ROS) or Python SDK, multiple Crazyflie 2.0 can be controlled simultaneously.

In the current setup, we equipped the nano UAVs with an optical motion detection system $(1.6 \mathrm{~g})$, a 3D local positioning system ( $3.3 \mathrm{~g})$, and a Sensirion SGP30 Multi-Pixel gas sensor. The resulting takeoff weight of one copter incl. battery is $35.6 \mathrm{~g}$, which will be further improved to gain longer flight times. Please see $[2,3]$ for further information.

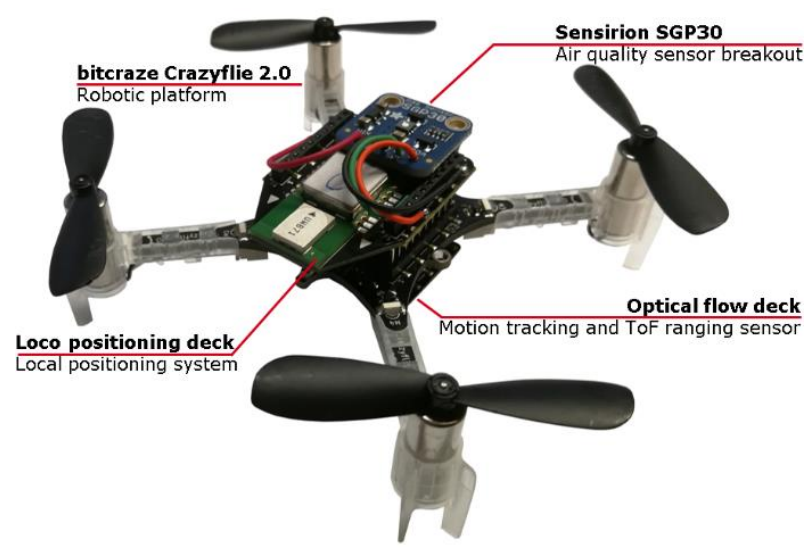

Fig. 1. The Crazyflie 2.0 quadrocopter platform with mounted sensors.

\section{Experimental Setup}

The time of flight sensor of the optical motion detection system measures the distance to the floor beneath a copter optically. Currently, objects - in this case a second copter - that move into the sensor's field of view are interpreted as the floor resulting in altitude leaps of the copter. To avoid this behavior, we removed the optical motion detection system of the upper flying copter in the experimental setup.

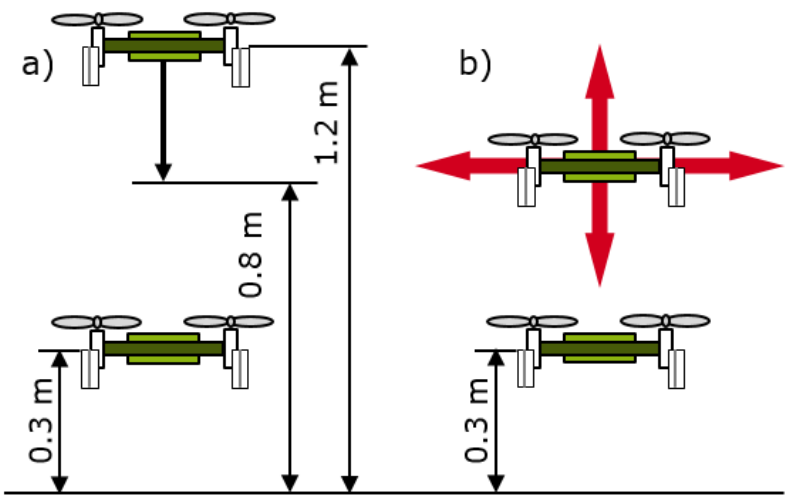

Fig. 2. Experimental setups of a) the minimum vertical distance and $b$ ) the horizontal/vertical displacement experiments. 


\subsection{Minimum Vertical Distance}

The experiment was performed with two nano UAVs - one hovering at an altitude of $0.3 \mathrm{~m}$ above the ground (CF1), while the second started to descent slowly from an altitude of $1.2 \mathrm{~m}$ to $0.8 \mathrm{~m}$ (CF4, see Fig. 2(a)). The experiment was stopped, once the flight behavior of $\mathrm{CF} 1$ become too unstable or CF1 finally crashed.

\subsection{Horizontal/Vertical Displacement}

Several flights with two nano UAVs were performed with varying the distance (vertically in $0.1 \mathrm{~m}$ and horizontally in $0.05 \mathrm{~m}$ steps) to each other, see Fig. 2(b). This was done to study the influence of the rotor downwind of a nano UAV flying above another. Again, the altitude of the hovering copter (CF1) was set to $0.3 \mathrm{~m}$, while the second copter (CF2) was flown to a defined position to examine the hovering performance of CF1. One experiment took $60 \mathrm{~s}$ to complete. As performance criteria, we used the combined standard deviation of $\mathrm{x}$, $\mathrm{y}$, and $\mathrm{z}\left(\sigma_{x y z}\right)$ of CF1 and the observations we made during the different trials.

\section{Experimental Results}

In total, we performed 77 trials: one reference trial with a horizontal offset of $1 \mathrm{~m}$ between the flying nano UAVs to determine the flight behavior of the copters independently from each other, three trials based on the setup described in Sec. 3.1, and the remaining 73 trials based on Sec. 3.2.

Fig. 3 shows exemplary the resulting trajectories of both nano UAVs of experiment 3.1. As soon as CF4 arrived at its starting position above CF1, the flight behavior of CF1 become more unstable resulting in staggering of the copter, which is comparable to the flight behavior of a copter without installed optical motion detection system. This staggering behavior of CF1 increased during descent of CF4. Finally, CF1 crashed when CF4 reached its minimum vertical distance to CF1 of $d_{\min }=0.5 \mathrm{~m}$.

The results of experiment 3.2 can be seen in Figs. 4 and 5. Based on the datasets obtained from the 73 trials, we calculated a map of $\sigma_{x y z}$, which is shown in Fig. 5. From this map, three main regions can be identified: a green region, where CF2 can fly without having a significant impact on the flight behavior of CF1 $\left(\sigma_{x y z}<0.045 \mathrm{~m}\right)$, an orange region, where a significant impact on CF1 can be observed $\left(0.045 \mathrm{~m} \leq \sigma_{x y z}<0.07 \mathrm{~m}\right)$, and a red region, where the rotor downwash of $\mathrm{CF} 2$ resulted in a crash of $\mathrm{CF} 1\left(\sigma_{x y z} \geq 0.07 \mathrm{~m}\right)$.

\section{Conclusion}

In this work, we investigated the influence of the rotor downwash on flying vertically displaced nano robots. From these experiments, we derived the minimum vertical distance between two copters of $0.5 \mathrm{~m}$ and a model describing a so-called safety region around each copter allowing a safe operation of the swarm indoors.

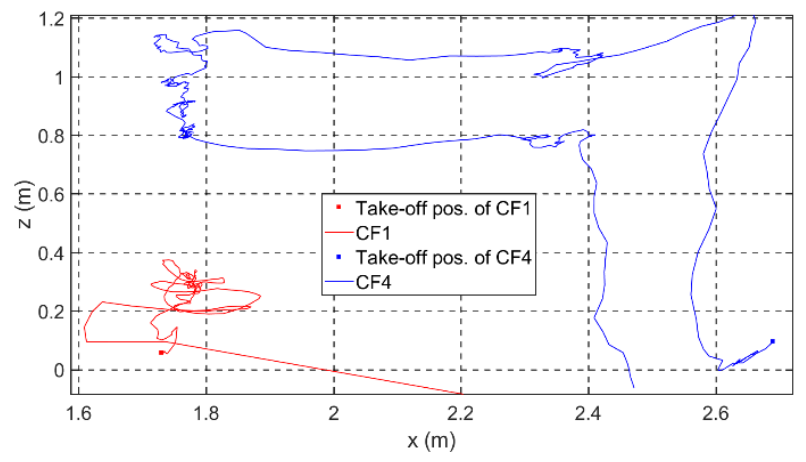

Fig. 3. Result of one vertically displaced flight.

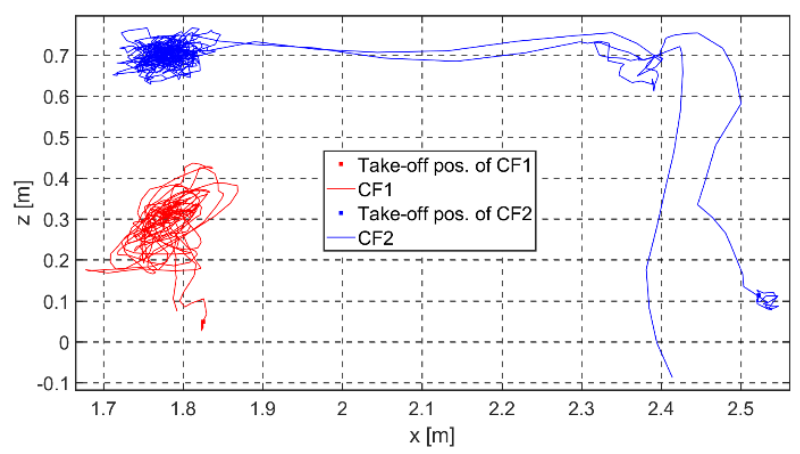

Fig. 4. Result of one flight with a horizontal/vertical displacement of CF2 of $0.15 \mathrm{~m}$ and $0.40 \mathrm{~m}$, respectively.

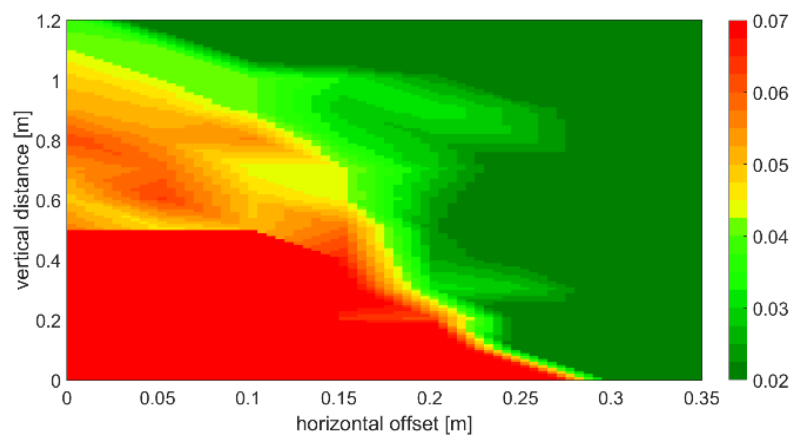

Fig. 5. 2D Map of the combined standard deviation $\sigma_{x y z}$ of the hovering copter CF1 in [m].

\section{References}

[1] https://www.bitcraze.io/

[2] Neumann, P. P., et al., 35th Danubia-Adria Symposium, Sinaia, Romania, 2018, pp. 139-140.

[3] Neumann, P. P., et al., 2019 ISOCS/IEEE International Symposium on Olfaction and Electronic Nose (ISOEN), Fukuoka, Japan, 2019, accepted. 